\title{
The RFID Technology for Neurosciences: Feasibility of Limbs' Monitoring in Sleep Diseases
}

\author{
Cecilia Occhiuzzi and Gaetano Marrocco, Member, IEEE
}

\begin{abstract}
This contribution investigates the feasibility of the passive UHF RF identification technology for the wireless monitoring of human body movements in some common sleep disorders by means of passive tags equipped with inertial switches. Electromagnetic and mechanical models as well as preliminary experimentations are introduced to analyze all the significant issues concerning the required power, the tag antenna design, the read distance, and the expected biosignals collected by the interrogation device.
\end{abstract}

Index Terms-Accelerometer, RF identification (RFID), sensor network, sleep disorder, tag antenna.

\section{INTRODUCTION}

$\mathbf{T}$ HE RESTLESS legs syndrome (RLS) and the periodic limb movements (PLMs) are common disorders affecting the sleep [1]. RLS is clinically characterized by a compelling urge to move the limbs, accompanied by uncomfortable and unpleasant sensations in the extremities, especially during periods of rest or inactivity such as lying or sitting. Symptoms of RLS show a characteristic circadian evolution with a nocturnal worsening leading to insomnia, and consequently, daytime sleepiness and reduced quality of life.

During the sleep, RLS is often associated with repetitive unilateral or bilateral limbs movements referred to as PLM, defined in terms of duration, number, and interval between movements. The movement is highly stereotyped and in its complete form resembles the so-called triple response: extension of the great toe followed by a partial flexion of the ankle, knee, and sometimes hip.

RLS is generally diagnosed by medical history while PLMs must be diagnosed by polysomnography (PSG) or movement recording [2]. The analysis requires the establishment of the number of leg movements that meet PLM criteria occurring during sleep and during wakefulness, the number of events leading to arousal, the duration of the intervals between successive movements, the duration of the movements themselves, and their occurrence.

At present [3], the most versatile diagnosis tool is the actigraph that embeds miniaturized accelerometers, a local memory, and a processing unit within a bracelet- or watch-like packaging. This device is usually placed on the ankle or foot and monitors local acceleration. In spite of the fact that it only provides motion information and cannot tell which muscle is generating the movement, the recorded data are useful for clinical purpose,

Manuscript received October 3, 2008; revised March 19, 2009. First published September 1, 2009; current version published January 15, 2010.

The authors are with the Dipartimento di Informatica, Sistemi e Produzione, University of Roma “Tor Vergata,” Rome 00133, Italy (e-mail: marrocco@ disp. uniroma2.it).

Digital Object Identifier 10.1109/TITB.2009.2028081 with the great advantage to conduct the analysis in the patients' home environment.

This paper discusses the feasibility of a completely new approach to monitor sleep disease, also suited to different motion analysis. The method is based on the emerging RF identification (RFID) technology and passive motion-detecting sensors. The purpose is to obtain very low-cost wireless devices, eventually single usage, able to be attached onto the body segments to be controlled, and to produce high-level aggregated data just ready for the clinical analysis.

An RFID system comprises two components: the remote transponder or tag, including an antenna and a microchip transmitter, located on the object to be identified, and the local querying system or reader, which can read and elaborate the data transmitted from the tag. Various kinds of data and first of all a unique identification (ID) code can be wireless-transferred to the reader by means of RF electromagnetic signals. RFID of objects and remote control of devices is becoming very popular in logistics, inventory management, and bioengineering applications [4]. A new frontier in this area is the wireless monitoring of people within mobile healthcare services [5], [6]. Due to the advances in low-power electronics, it is now feasible to envisage sophisticated RFID-like devices integrating sensing and signal processing [7] able to provide real-time biomonitoring (temperature, blood pressure, heartbeat, glucose content, human behavior) and tracking of a person's movement within hospitals or domestic environments.

The tags could be passive, harvesting energy from the interrogating system, semiactive when a battery is included only to feed the sensors, or fully active where a local source directly feeds a microcontroller as well as the transmitting radio. Focusing on passive systems, which may have a near unlimited life and very low cost, the most attractive standard is the UHF (860-960 MHz), which, in principle, promises a significative activation range. However, the design of passive UHF RFID antennas for on-body applications is still an open issue since the presence of the human body greatly degrades the radiation and reading performances. In addition, the design of UHF RFID antennas involves conjugate impedance matching to microchip transponders having high capacitive input reactance. In a recent paper [8], the design of UHF RFID planar antennas placed onto the human body has been considered and a tag antenna configuration of simple geometry, suited to host sensors, has been introduced.

The application of the RFID technology to the monitoring of limb movements (see a possible setup in Fig. 1) during the sleep is a rather new topic. It requires the integration of passive inertial switches over the tag antenna, as in the enhanced passive UHF RFID proposed in [9] and [10], and also presents 


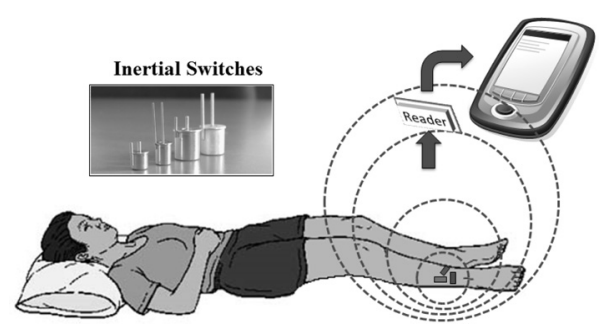

Fig. 1. Possible setup for the wireless monitoring of limbs' motion by RFID technology equipped with passive motion-detecting sensors.

the great challenge to design passive UHF tags suited to the human body. The paper considers the power sensitivity of the microchip transponder, the tag antenna design, the sensitivity and the time response of the inertial switch, the emitted electromagnetic power from the reader and the interrogation rate with respect to the read distance, and the retrievable information about motion.

This study is carried on by means of numerical simulations based on both electromagnetic and mechanical tools and preliminary experimental validations.

The paper is organized as follows. Section II analyzes in detail the issues related to the key parameters of the restless sleep monitoring, the powers, the timing, and the achievable read distance. The design of body-matched tag and its preliminary experimental characterization are presented in Section III while Section IV includes the discussion on the estimated biophysical signals. Finally, the concluding remarks and some possible variations of the platform are given in Section V.

\section{System ANALYSIS}

The proposed monitoring method requires one or more UHF RFID tags equipped with inertial switches to be attached onto the patient's limbs or onto other body regions. As proposed in [9] and [10], the switches react to the verse of the applied acceleration by changing their internal impedance from low (ideally, short circuit) to high (ideally, open circuit) impedance. A wireless one-axis sensor may then be achieved by means of a tag embedding two reverse-oriented inertial accelerometers (for instance, see the scheme in Fig. 4), which turn between two RFID microchip transponders so that only one of the two possible IDs is emitted. Such an ID may be related to the acceleration's verse according to an ID modulation paradigm, as described more in details in Section IV.

The tags are interrogated, according to a proper repetition rate, by an RFID reader placed somewhere in the patient's rooms, for instance, on the sidewalls or, better, on the ceiling. The feasibility of this configuration is strictly correlated to the correct interrogation of the tags from a given distance in accordance with the safety exposure regulations.

In this perspective, it is important to define: 1) the key parameters of the restless sleep monitoring; 2) the minimum features required for the body-antenna design; 3) the number and the position of the tags; 4) the sensibility of the inertial switches; and 5) the energetic and safety constraints. These issues are now analyzed in detail in the following paragraphs.

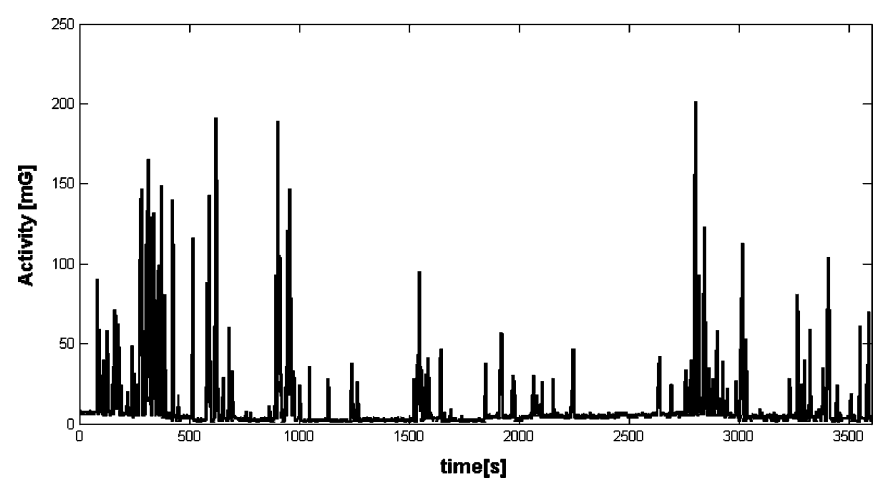

Fig. 2. Typical actigraphic trace for PLM reporting the amplitude of the acceleration during $1 \mathrm{~h}$ time interval and sampled at $f_{C}=1 \mathrm{~Hz}$. Courtesy Neuroscience Laboratory, University General Hospital of Roma "Tor Vergata."

\section{A. Restless Sleep Key Parameters}

PLMs are well defined in terms of duration, number, and interval between movements. According to the operational definition of PLMs given by the World Association of Sleep Medicine (WASM) [11], the duration of a typical PLM event is about $0.5-5 \mathrm{~s}$ and the time elapsed between two consecutive events to be considered as PLM must be at least $5 \mathrm{~s}$ and no more than $90 \mathrm{~s}$. Movements must occur in a sequence of required length, and the number of consecutive candidate events matching the period criteria must be four in number or more. As suggested in [11], the most relevant parameters to monitor are the time duration of each PLM and their repetition rate all along the night. Fig. 2 shows an example of actigraphic data during an episode of sleep disorder. It has been experimentally found that the quiet-state (normal sleep) acceleration is about $5 \mathrm{mG}$ (with $G=9.81 \mathrm{~m} / \mathrm{s}^{2}$ ), while during a PLM, the acceleration may reach an average value of $0.2 \mathrm{G}$. The WASM standards indicate that the dynamics required for the acceleration sensors are [0.01$8 \mathrm{G}]$. The same recommendation suggests a sampling rate of the motion capturing of $f_{c} \geq 10 \mathrm{~Hz}$, and typically, the conventional actigraphs consider two data sampling rates at 1 and $16 \mathrm{~Hz}$, respectively.

\section{B. Tag Sensitivity and Useful Read Region}

Since the proposed sensing platform is based on the RFID technology, some basic definitions are now quickly recalled.

At the beginning of the reader-to-tag communication protocol [12], the reader first activates the tag, placed over a target object, by sending a continuous wave, which, by charging an internal capacitor, provides the required energy to perform actions. During this listeningmode, the microchip exhibits an input impedance $Z_{\text {chip }}=R_{\text {chip }}+j X_{\text {chip }}$, with $X_{\text {chip }}$ being capacitive. The antenna impedance $Z_{A}=R_{A}+j X_{A}$ has to be matched to $Z_{\text {chip }}\left(Z_{A}=Z_{\text {chip }}^{*}\right)$ for maximum power transfer. The fraction $P_{R \rightarrow T}$ of the reader input power that is absorbed by the tag is

$$
P_{R \rightarrow T}=\left(\frac{\lambda_{0}}{4 \pi r}\right)^{2} G_{R}(\theta, \phi) G_{T}(\theta, \phi) \tau P_{\mathrm{in}} \chi
$$


where $\lambda_{0}$ is the free-space wavelength, $r$ is the reader-tag distance, $G_{R}$ is the gain of the reader antenna, $G_{T}$ is the gain of the tag's antenna placed on the target, $P_{\text {in }}$ is the RF power provided to the reader antenna, and $\tau$ is the power transmission coefficient of the tag, which is defined as

$$
\tau=\frac{4 R_{\text {chip }} R_{A}}{\left|Z_{\text {chip }}+Z_{A}\right|^{2}}
$$

and $\chi$ is the polarization efficiency between the reader and the tag, generally close to $1 / 2$ provided that reader adopts a circularly polarized antenna, as it is usual. The tag is activated when the absorbed power exceeds the tag's microchip sensitivity threshold $p_{T}: P_{R \rightarrow T}>p_{T}$. From (1), the activation region of the reader, e.g., the places $\Omega_{R}$ where a tag collects enough energy to be activated, is

$$
\Omega_{R}=\left\{\underline{r}: \frac{P_{R \rightarrow T}}{p_{T}}>1\right\}
$$

and the maximum activation distance $r_{R}$ for the tag is computed from (1) by enforcing $P_{R \rightarrow T}=p_{T}$

$$
r_{R}=\max _{\theta, \phi}\left\{\frac{\lambda_{0}}{4 \pi} \sqrt{G_{R}(\theta, \phi) \hat{G}_{T}(\theta, \phi) \frac{P_{\mathrm{in}}}{p_{T}}} \chi\right\} .
$$

The function $\hat{G}_{T}=G_{T} \tau$ is the realized gain of the tag's antenna, when it is placed onto the body, and it is one of the most important performance parameter of the tag.

During the next steps of the communication, the tag receives the command coming from the reader and finally sends back the data through a backscattered modulation of the continuous wave coming from the reader itself. In this case, the tag's IC acts as a programmable switching device between a low impedance and a high impedance, thus modifying the reflectivity of the tag responding, and hence the strength of the reflected power.

Going back to the considered application, the limb-worn tags have to be read within a typical hospital or a domestic room under the constrain that the emitted field is compliant with the specific exposure limits. It is hence useful to introduce the concept of forbidden region, corresponding to a particular reader emission, as the place where the rms electric field (averaged over a prescribed time interval $T_{\mathrm{av}}$ ) is higher than the maximum rms value $E_{0}$ allowed by the local safety regulations. In general, the reader can interrogate the environment according to a given repetition period $T_{0}$. Denoted with $T_{\text {com }}$ the time duration of a typical reader-tag communication, the duty cycle is $d=T_{\text {com }} / T_{0}$. The forbidden region is therefore

$$
\Omega_{F}=\left\{\underline{r}: \frac{\langle E(r)\rangle_{T_{\mathrm{av}}}}{E_{0}}>1\right\}
$$

where $\left\langle E(r)_{\mathrm{rms}}\right\rangle_{T_{\mathrm{av}}}=\sqrt{d} E(r)_{\mathrm{rms}}$ is the rms time-averaged reader's field.

The boundary $r_{F}(\theta, \phi)$ of $\Omega_{F}$ can be predicted with good accuracy by just assuming free-space propagation, e.g.,

$$
r_{F}(\theta, \phi)=\sqrt{30 P_{\text {in }} G_{R}(\theta, \phi) d} / E_{0}
$$

with $E_{0}$ in given rms unit, for instance, $E_{0}=1.375 \sqrt{f}=$ $41.3 \mathrm{~V} / \mathrm{m}$ according to the European Recommendation [13].
Even more restrictive constraints may be found in some countries, as in the case of Italy where $E_{0}=6 \mathrm{~V} / \mathrm{m}$.

The electric parameters are, therefore, fixed so that the useful region for the patient is the hollow volume $\Omega_{U}=\Omega_{R}-\Omega_{F}$. Restrictive exposure limits impose the use of a reduced emitted power (small forbidden region) and rather sensitive microchips (large read region), for instance, by designing tags with realized gain $\hat{G}_{T}=0 \mathrm{~dB}$ and sensitivity $p_{T}=30 \mu \mathrm{W}(-15 \mathrm{dBm})$ and by using a reader emitting 3.2 W EIRP (emission limit in Europe) in continuous mode $(d=1)$; hence, $r_{R}=6 \mathrm{~m}$ and $r_{F}=0.2 \mathrm{~m}$ $\left(E_{0}=41.3 \mathrm{~V} / \mathrm{m}\right)$. The useful region will, therefore, have maximum extension $r_{R}-r_{F}=5.8 \mathrm{~m}(4.5 \mathrm{~m}$ for the more restrictive limit $E_{0}=6 \mathrm{~V} / \mathrm{m}$ ), which is reasonable to include a sleeping room. A worse performing tag's antenna, as expected for body tags, needs to be compensated by a more sensitive chip.

\section{Timing}

By considering that the limb's motion is required to be sampled at rate $f_{c}$, the number of RFID microchips that may be interrogated are given by

$$
N_{C}=\frac{d}{f_{c} T_{\text {com }}} .
$$

Under the assumptions that $f_{c}=10 \mathrm{MHz}$, a typical duration of a reader-tag interrogation $T_{\text {com }}=30 \mathrm{~ms}$, and finally, a duty cycle $d=1, N_{C}=3.3$. Since only one of the two microchips placed in the tag will be responding (depending on the acceleration verse), the system timing will, therefore, permit to acquire the information of three tags that could be placed either onto a same limb to collect the three-axis motion pattern, or onto different body segments such as the other limb and the thorax. Some realistic simulated examples will later on demonstrate that even a single-axis monitoring will still provide useful overall data for medical considerations.

An inertial switch changes its impedance state in reply to a motion stimulus after a delay time $t_{\text {switch }}$, which has to be smaller than the interrogation period $1 / f_{c}=0.1 \mathrm{~s}$. Typical commercial devices [14] show values $t_{\mathrm{switch}} \simeq 0.01 \mathrm{~s}$, which are hence compatible with the considered application.

\section{Body TAG DESIGN}

The antenna of RFID tag is the real critical issue of the proposed platform. Designing UHF tag antennas for on-body application is generally a hard goal since the presence of the human body causes a strong pattern distortion and efficiency degradation due to dissipation and scattering. As in [8], among the many available options for the RFID tag design [15], the planar slot family is considered here since it can easily host additional electronics at both sides of the antenna, and hence, it is also suited to include contacting sensors. The basic geometry consists of an $\mathrm{H}$-shaped slot cut over a square suspended patch attached onto the human body through an insulating material. The patch size mainly governs the gain, and hence the maximum read distance, while the aspect ratio of the slot affects the impedance tuning. In [8], many examples about the possibility to match both lowimpedance and high-impedance microchips are reported. The 


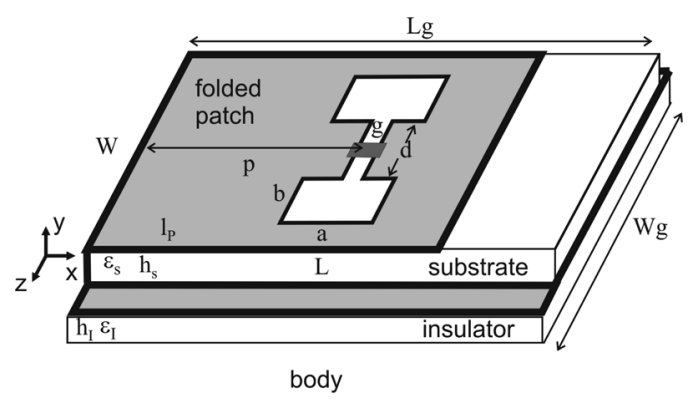

Fig. 3. Layout of the proposed tag family. The $\mathrm{H}$-slot acts as tuning impedance. The sensors may be allocated over the top conductor.

critical issue with this antenna is the low gain as found in many on-body tag examples (estimated maximum values of $-10 \mathrm{~dB} \div$ $-7 \mathrm{~dB}$ in the best geometries). In the specific context, however, the motion sensors, e.g., the inertial switches, do not need to be placed in electric contact with the body, and hence, the aforementioned structure can be modified with the purpose to better isolate the radiating part of the antenna from the body, reducing radiation losses into the body districts where the tag is placed, and thus improving the gain. The enhanced wearable antenna is depicted in Fig. 3. The rectangular plate has been folded around a dielectric slab of height $h_{S}$ and the longest face is placed over the body through an optional dielectric insulator slab of thickness $h_{I}$. This geometry can be viewed as a series-fed "L" patch [unlike the shunt-fed conventional planar inverted-F antenna (PIFA)], e.g., as a two-plate transmission line, terminated at the left end by a nonideal short circuit, by a nonideal open circuit at the right end, and partly loaded with a coupled discontinuity (the upper slot). In order to better isolate the antenna from the body, the lower plate is slightly wider than the upper one $\left(W_{g}>W\right.$ and $\left.L_{g}>L\right)$, thus reducing the dissipation in body tissues.

The radiation can be related to the left folding and mostly to the slot and the right truncation. The polarization is linear and parallel to the antenna's main direction ( $x$-axis in the figure). Assuming that the thickness $h_{S}$ of the inner dielectric is small compared with the wavelength, the radiation from the folding may be considered negligible and the gain and matching features of the antenna may be related only to the slot and the transmission line truncation.

As for conventional patches, the increase in the horizontal size $W$ produces a gain enhancement. Depending on the position of the tag over the body and hence on the available space, it is possible to increase the dimension in order to achieve better radiation performances. The length $L$ of the patch is chosen approximately equal to $\lambda / 4$, where $\lambda$ is the effective wavelength in the dielectric substrate.

A possible packaging of the wearable tag with the inertial switches is sketched in Fig. 4, where the arrows indicate the activation verse of the switch.

\section{A. Tag Prototype}

An early prototype has been designed by using a finitedifference time-domain (FDTD) computer tool [16] that also accounts for the presence of the human body by means of a strat-

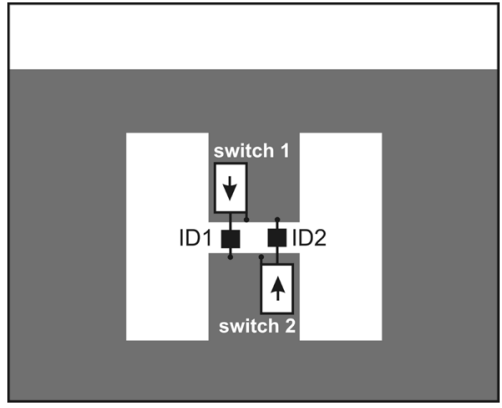

Fig. 4. Example of integration between the wearable antenna (front view), the inertial switches (white boxes), and two microchips (black boxes). The arrows indicate the preferred direction of the switches.

TABLE I

PhysicAl AND GEOMETRICAL PARAMETERS OF THE SiMPLIFIED LiMB MODEL AT $870 \mathrm{MHz}$

\begin{tabular}{|c|c|c|c|}
\hline Layer & $\varepsilon_{r}$ & $\begin{array}{c}\sigma \\
{[S / m]}\end{array}$ & $\begin{array}{c}\text { Cross } \\
\text { section }[\mathrm{cm}]\end{array}$ \\
\hline Skin + fat & 14.5 & 0.25 & $12 \times 11$ \\
\hline Muscle & 55.1 & 0.33 & $10 \times 9$ \\
\hline Bone & 20.8 & 0.33 & $4 \times 2$ \\
\hline & \\
& \\
&
\end{tabular}

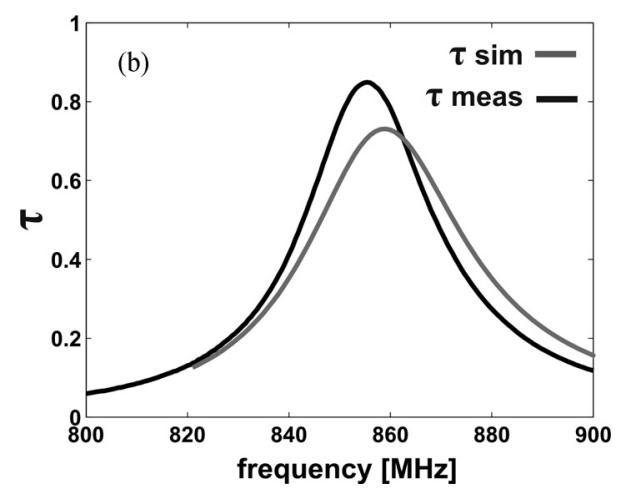

Fig. 5. (a) Fabricated prototype of body-worn tag matched to $Z_{\text {in }}=15-$ $j 135 \Omega$ microchip. Size in millimeters. $L=49, L_{g}=60, W=W_{g}=60$, $a=8, b=10, p=L / 2, d=10, g=3, h_{S}=4$, and $h_{I}=2$. (b) Simulated and measured power transmission coefficient.

ified box model of height $40 \mathrm{~cm}$ (parameters in Table I), whose sizes resemble a limb. The polytetrafluoroethylene (PTFE, popularly known as Teflon: $\varepsilon_{S} \equiv \varepsilon_{1}=2.08, \tan \delta=4 \times 10^{-4}$ ) has been used as both an inner and an external dielectric, while the conductors are made up of adhesive copper sheets. The prototype is shown in Fig. 5(a) and is matched to a microchip with input impedance $Z_{\text {chip }}=15-j 135 \Omega$. A measurement of tag impedance verified a better than $70 \%$ power transmission coefficient at $869 \mathrm{MHz}$ and good agreement with simulated data [Fig. 5(b)]. 


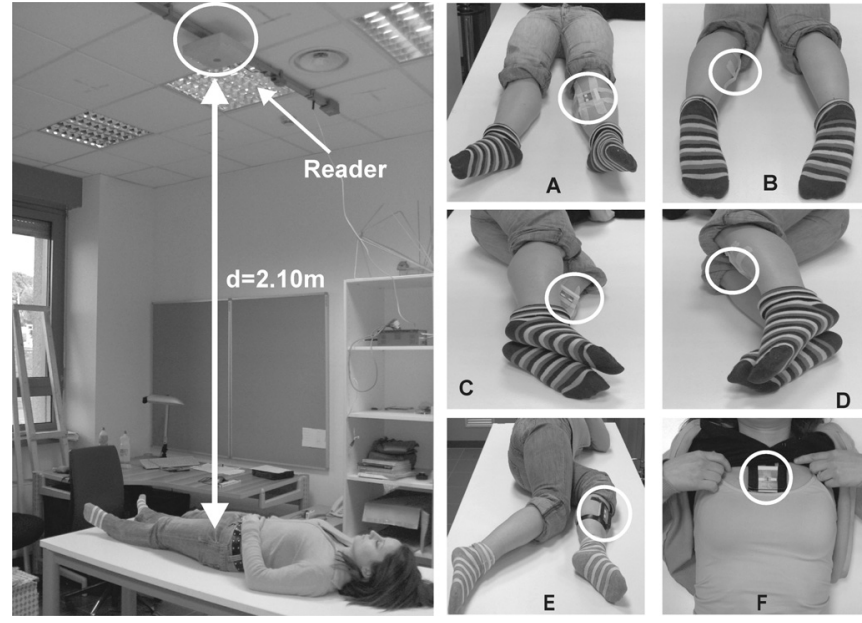

Fig. 6. (Left) Measurement setup comprising the long-range reader, the table, and the patients. (Right) Typical sleep positions (a)-(f). (a)-(e) Antenna is placed in front of the left leg and (f) on the thorax.

\section{B. Performances Over Body in Real Configurations}

Since the tag antenna has to guarantee the correct reader-tag communication during the whole sleep, when the body pose may change many times, it is important to analyze the prototype's performances in real configurations. Among typical sleep positions [17], the most threatening ones are those with joined legs since the tag could be subjected to higher losses and pattern distortion. The worst condition arises when the tag is sandwiched by the two arms and it may reasonably occur only if the tag is placed in the inner part of the leg. Therefore, it is opportune to place the tag onto the front or external surface.

The effective communication properties of the proposed system have been evaluated by using the setup in Fig. 6, comprising a long-range, remotely controlled reader CAEN A948, and a circular polarized patch antenna with $8 \mathrm{~dB}$ maximum gain. The measurement setup reproduces a typical medical scenario: a bed and a properly spaced RFID reader, e.g., placed on the ceiling. To better understand the antenna performance independently on the particular embedded sensor, the tag has been experimented without inertial switches, whose full characterization and integration requires further research. In successive measurements, the tag is placed on the middle of the left leg (in frontal position) and on the center of the thorax of a volunteer, and has been interrogated under typical sleep positions [17]. For each body pose, the reader records the minimum effective isotropically radiated power (EIRP $=P_{\mathrm{in}} G_{R}$ ) required to activate the tag and collect a stable response. This is a useful indicator to discuss the feasibility of this system and to get an idea about the power margin to increase the read distance in larger environments.

Table II summarizes the activation EIRP and the estimated effective electric field $\left\langle E(r)_{\mathrm{rms}}\right\rangle_{T_{\mathrm{av}}}$ from (6) corresponding to the patient. The tag is efficiently readable in each case, even in the back position (B) and in presence of the second leg (C and D). The required activation powers and the relating fields are perfectly in accordance with the European Recommendations clarified in Section II, and even with the more penalizing Italian
TABLE II

EIRP OF THE READER AND EFFECTIVE ELECTRIC FIELD CORRESPONDING TO THE PATIENT

\begin{tabular}{|c|c|c|}
\hline Position & $E I R P[W]$ & $\langle E(r)\rangle_{\text {r.m.s }}[V / m]$ \\
\hline A & 2.34 & 3.99 \\
\hline B & 1.97 & 3.66 \\
\hline C & 3.20 & 4.67 \\
\hline D & 3.02 & 4.53 \\
\hline E & 3.20 & 4.67 \\
\hline F & 1.24 & 2.90 \\
\hline
\end{tabular}

one. The minimum required EIRP corresponds to the tag placed on the torso, while the maximum power, as expected, is referred to the tagged leg partially covered by the other one. This pose is, however, not so common during the PLM episode since movements can be monolateral or bilateral, and the patient generally moves the legs separately. In a typical medical scenario, a $3.2 \mathrm{~W}$ EIRP would be more than enough to monitor the patient during all along the sleep, regardless his positions and his movements.

It is important to report that simulations and tests have demonstrated that the antenna performances are adequate to the position of the tag on different body segments, due to the conductor folding, which decouples the radiating part of the antenna from the tagged body. Such a tag is, therefore, a good candidate for the monitoring of other body motion patterns.

\section{Estimated BiophysicAl Signals By USING A RADIOMECHANICAL MODEL}

Assuming now that the reader-tags links may be reliable during all along the sleep, a radiomechanical model is introduced here to simulate the biophysical signals collected by the reader during the legs motion and to discuss the effect of other system parameters such as the inertial switch's threshold and the interrogation rate. The model includes a simplified human phantom having individually controllable 18 moving parts. By a preliminary analysis of maximum articular flexion and extension of the legs' segments, some typical motion patterns in PLM episodes have been reconstructed by enforcing the trajectory of hip, knee, and foot. The movement has been computed by a multibody cinematic simulation solver [18].

The numerical computation produces the vectorial acceleration at any part of the body. A four-event PLM episode (Fig. 7), occurring within an interval of $50 \mathrm{~s}$, has been simulated. Since the considered events are flexions and extensions of ankle and knee around the transversal axis ( $z$-axis), the motions take place only on $x-y$ plane, and hence, only two tags are supposed to be used. Fig. 8(a) gives the predicted acceleration of the leg at tags positions.

The one-axis accelerometer is simply modeled as an ideal switch changing between a short circuit and a high-impedance $Z_{O C}$ (ideally, an open circuit), depending on the applied acceleration. The instantaneous transfer function of the switch included in the model is

$$
Z_{S}^{\mp}\left(a_{\xi}\right)=Z_{H} U\left(a_{S} \pm a_{\xi}\right)
$$

where $\xi$ indicates a component of the applied acceleration, $Z_{S}^{\mp}$ is the impedance of the switch oriented toward $\xi^{-}$or $\xi^{+}, U(\cdot)$ 


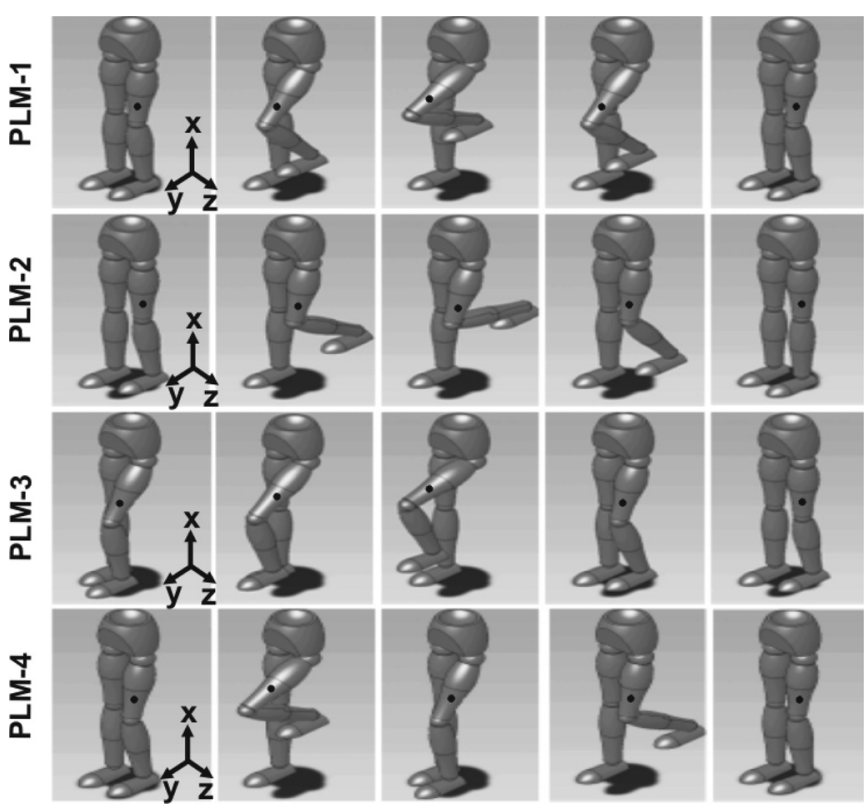

Fig. 7. Motion patterns for four PLM episodes. To speed up the cinematic computations, only the lower part of the phantom has been considered.

(a)

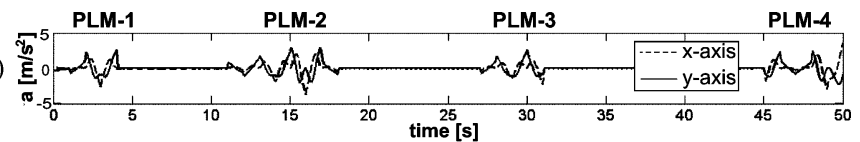

(b)

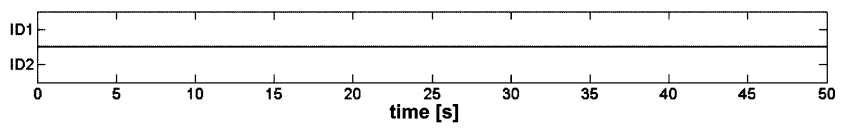

(c)

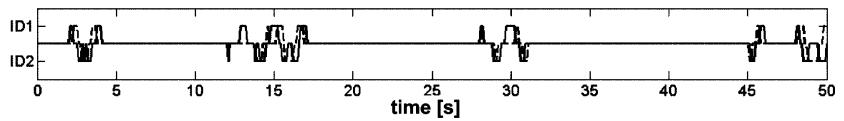

(d)

Fig. 8. (a) Leg's acceleration and ID-modulated received data for sampling rate $f_{C}=10 \mathrm{~Hz}$ and three different acceleration thresholds. (b) $a_{s}=0.5 \mathrm{G}$. (c) $a_{s}=0.1 \mathrm{G}$. (d) $a_{s}=0.01 \mathrm{G}$.

is the Heaviside function, and $a_{S}$ is the threshold acceleration. This model does not account for the switch's inertial delay since typical values of commercial device, as discussed in Section III$\mathrm{B}$, are much smaller than the duration of PLM episodes. The presence of the tag is accounted such that when the leg is moving toward the positive $\xi^{+}\left(a_{\xi}>a_{S}\right)$, the switch 2 will be "closed" connecting the microchip 2 to the antenna port, while the switch 1 will be open, and hence, the reader will collect the only ID2 code. The converse holds in case of $a_{\xi}<-a_{S}$.

The ID-modulated data received by the reader are shown in Figs. 8 and 9 for different choices of the acceleration threshold $a_{S}$ and the interrogation frequency $f_{C}$. The particular choice of the parameter $a_{S}$ affects the detection of low movements.

Interrogation at frequency $f_{C}=1 \mathrm{~Hz}$ cannot correctly reproduce PLM movements (Fig. 9), while having chosen $f_{C}=5 \mathrm{~Hz}$, it is instead possible to collect useful data about the number of PLM, their duration, and the time elapsed between two con-

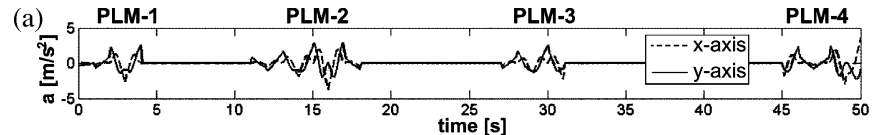

(b)

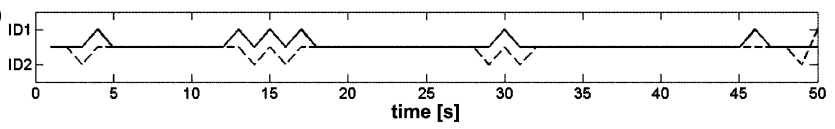

(c)

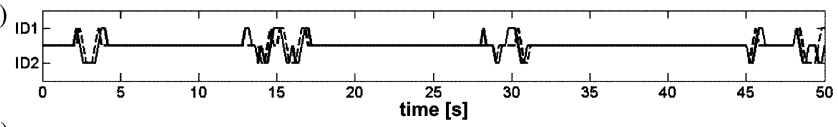

(d)

Fig. 9. (a) Leg's acceleration and ID-modulated received data for switch's acceleration threshold $a_{s}=0.1 \mathrm{G}$ and some interrogation frequency. (b) $f_{C}=$ $1 \mathrm{~Hz}$. (c) $f_{C}=5 \mathrm{~Hz}$. (d) $f_{C}=10 \mathrm{~Hz}$.

TABLE III

ESTIMATED PLM DURATION FOR DIFFERENT SWITCH THRESHOLD AND INTERROGATION RATE

\begin{tabular}{|c|c|c|c|c|c|}
\hline \multirow{2}{*}{ PLM } & \multirow{2}{*}{$T_{P L M}$} & \multicolumn{2}{|c|}{$f_{c}=5 H z$} & \multicolumn{2}{|c|}{$f_{c}=10 H z$} \\
\hline & & $0.1 G$ & $0.01 G$ & $0.1 G$ & $0.01 G$ \\
\hline \multirow[b]{2}{*}{1} & \multirow{2}{*}{$4.1 \mathrm{~s}$} & $T_{x}=2.4$ & 4.0 & 2.5 & 4.0 \\
\hline & & $T_{y}=3.0$ & 4.2 & 3.0 & 4.0 \\
\hline \multirow{2}{*}{2} & \multirow{2}{*}{7.0} & 4.0 & 6.8 & 4.0 & 6.8 \\
\hline & & 5.2 & 7.0 & 6.0 & 7.0 \\
\hline \multirow{2}{*}{3} & \multirow{2}{*}{4.0} & 3.2 & 4.0 & 2.5 & 3.8 \\
\hline & & 3.8 & 4.0 & 3.8 & 4.0 \\
\hline \multirow{2}{*}{4} & \multirow{2}{*}{5.5} & 5.0 & 5.0 & 4.7 & 5.0 \\
\hline & & 5.0 & 5.0 & 5.0 & 5.1 \\
\hline
\end{tabular}

secutive events. At this frequency, it is also possible, from (7), to interrogate up to $N_{C}=6$ tags, and hence to earn three-axis information in two distinct body regions.

To better discuss these results, a temporal index for the PLM, e.g., the time duration of each PLM, has been computed as the time interval between the first and the last ID transition of each PLM. With reference to Table III, it can be observed that:

1) each tag is able to correctly capture the dynamics in term of number of predicted PLM frequency and approximate duration;

2) a simple one-axis tag seems to be enough to capture the relevant features of the physical phenomenon;

3) an acceleration threshold $a_{S}=0.1 \mathrm{G}$ is enough to give an approximate estimation of the event duration.

Some commercial devices [14] show values $a_{S}=0.1 \mathrm{G}$, which are hence compatible with the considered application.

Alternatively, in order to detect just the motion of the legs without information about its pattern, it is possible to consider a single tag equipped with an omnidirectional accelerometer instead of three tags with a couple of reverse-oriented unidirectional switches. In this case, the sensor activates the tag response for any movement, and the two events collected by the reader will be null ID (motionless leg) and tag's ID (leg in motion) or vice versa. This is a simplified and a much less costly version of the proposed system useful for the diagnosis of all the neuromuscular diseases that require the detection of the movement more than its evolution. 


\section{DisCUSSIONS AND CONCLUSION}

This study theoretically demonstrates that the RFID monitoring of human motion, and in particular of the legs in sleep diseases, seems to be feasible within the actual technology and regulation constraints. A new tag geometry has been presented and tested in real configurations. The proposed antenna permits to host the motion sensors and seems to be a good candidate for monitoring people in conventional indoor area.

Using only passive components, it possible to obtain very lowcost wireless devices, eventually single usage. The tag could be printed on flexible substrates and attached onto the body segments by standard band-aids.

Moreover, the proposed monitoring strategy has various degrees of freedom, which may be used to collect further information about the patient's state during the night, such as position and complex movements, by using additional tags equipped with inertial sensors placed on his torso or shoulder. This is a supplementary feature of the proposed system that can help clinicians making differential diagnosis of the disease.

\section{ACKNOWLEDGMENT}

The authors wish to thank L. Bianchi, F. Placidi, and A. Romigi of the Neuroscience Department at the University of Roma Tor Vergata for suggestions and valuable discussions. They would also like to thank S. Cippitelli for his relevant contributions to this research.

\section{REFERENCES}

[1] M. J. Thorpy, Handbook of Sleep Disorders. New York: Informa Healthcare, 1990.

[2] W. Hening, "The clinical neurophysiology of the restless legs syndrome and periodic limb movements. Part I: Diagnosis, assessment, and characterization," Clin. Neurophysiol., vol. 115, pp. 1965-1974, 2004.

[3] S. Ancoli Israel, R. Cole, C. Alessi, M. Chambers, W. Moorcroft, and C. P. Pollak, "The role of actigraphy in the study of sleep and circadian rhythms," Sleep, vol. 26, no. 3, pp. 342-392, May 2003.

[4] K. Finkenzeller, RFID Handbook. New York: Wiley, 2000.

[5] B. Lo, "Key technical challenges and current implementations of body sensor networks," in Proc. Int. Workshop Wearable Implantable Body Sens. Netw., Apr. 12-13, 2005, pp. 1-5.

[6] M. Philipose, K. P. Fishkin, M. Perkowitz, D. J. Patterson, D. Fox, H. Kautz, and D. Hahnel, "Inferring activities from interactions with objects," IEEE Pervasive Comput., vol. 3, no. 4, pp. 50-57, Oct.-Dec. 2004.

[7] S. Nambi, S. Nyalamadugu, S. M. Wentworth, and B. A. Chin, "Radio frequency identification sensors," Proc. 7th World Multiconf. Syst., Cybern. Inf. (SCI 2003), pp. 386-390.

[8] G. Marrocco, "RFID antennas for the UHF remote monitoring of human subjects," IEEE Trans. Antennas Propag., vol. 55, no. 6, pp. 1862-1680, Jun. 2007.

[9] J. R. Smith, B. Jiang, S. Roy, M. Philipose, K. Sundara-Rajan, and A. Mamishev, "ID modulation: Embedding sensor data in an RFID timeseries," presented at the 7th Inf. Hiding Workshop, Barcelona, Spain, Jun. 6-8, 2005.

[10] A. P. Sample, D. J. Yeager, P. S. Powledge, and J. R. Smith, "Design of a passively-powered, programmable sensing platform for UHF RFID systems," in Proc. IEEE Int. Conf. RFID, Grapevine, TX, Mar. 26-28, 2007, pp. 149-156.
[11] M. Zucconi, R. Ferri, R. Allen, P. C. Baier, and O. Bruni, "The official world association of sleep medicine (WASM) standards for recording and scoring periodic leg movements in sleep (PLMS) and wakefulness (PLMW) developed in collaboration with a task force from the international restless legs syndrome study group (IRLSSG)," Sleep Med., vol. 7, pp. 175-183, 2006.

[12] P. V. Nikitin and K. V. S. Rao, "Theory and measurement of backscattering from RFID tags," IEEE Antennas Propag. Mag., vol. 48, no. 6, pp. 212 218, Dec. 2006.

[13] (1999, Jul. 30). European Council Recommendation 1999/519/EC of 12 July 1999 on the limitation of exposure of the general public to electromagnetic fields (0 Hz to $300 \mathrm{GHz}$. Official J. [Online]. L199, p. 59. Available: http://ec.europa.eu/enterprise/electr_equipment/lv/rec519.pdf

[14] Inertia Switch ${ }^{\circledR}$ : Miniature series acceleration switches. (2007). [Online] Available: http://www.inertiaswitch.com

[15] G. Marrocco, "The art of UHF RFID antenna design: Impedance-matching and size-reduction techniques," IEEE Antennas Propag. Mag., vol. 50, no. 1, pp. 66-79, Feb. 2008.

[16] G. Marrocco and F. Bardati, "BEST: A finite-difference solver fo time electromagnetics," Simul. Pract. Theory, vol. 7, no. 7, pp. 279-293, 1999.

[17] M. Y. Agargun, M. Boysan, and L. Hanoglu, "Sleeping position, dream emotions, and subjective sleep quality," Sleep Hypnosis, vol. 6, no. 1, pp. 8-13, 2004.

[18] SolidWorks Corporation, Concord, MA. COSMOSMotion user's manual (2006). [Online]. Available: http://www.solidworks.it

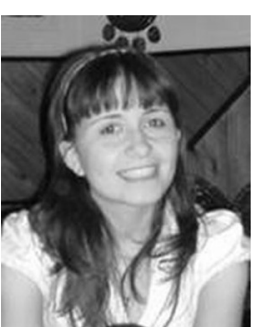

Cecilia Occhiuzzi was born in Paola, Italy, in 1983. She received the M.Sc. degree in medical engineering from the University of Rome "Tor Vergata," Rome, Italy, where she is currently working toward the Ph.D. degree in geoinformation.

During 2008, she was a Postgraduate Student in the School of Engineering, University of Warwick, U.K. Her current research interests include wireless health monitoring by means of RF identification and ultrawideband techniques.

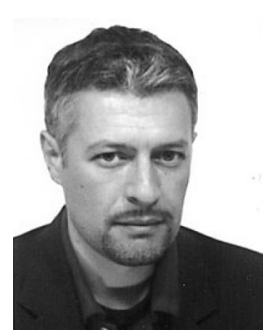

Gaetano Marrocco (M'98) was born in Teramo, Italy, on August 29, 1969. He received the Laurea degree in electronic engineering and the Ph.D. degree in applied electromagnetics from the University of L'Aquila, L'Aquila, Italy, in 1994 and 1998, respectively.

Since 1997, he has been a Researcher at the University of Rome "Tor Vergata," Rome, Italy, where he currently teaches antenna design and bioelectromagnetics. During summer 1994, he was a Postgraduate Student at the University of Illinois at Urbana Champain. During autumn 1999, he was a Visiting Scientist at Imperial College, London. His current research interests include modeling and design of broadband and ultrawideband antennas and arrays as well as of miniaturized antennas for RF identification (RFID) applications. He has been involved in several space, avionic, naval, and vehicular programs of the European Space Agency, North Atlantic Treaty Organisation (NATO), Italian Space Agency, and the Italian Navy. He holds two patents on broadband naval antennas and one patent on sensor RFID systems.

Dr. Marrocco is currently an Associate Editor of the IEEE ANTENNAS AND WIRELESS PROPAGATION LETTERS. 